therapy, $22 \%$ with NSAIDs and $96 \%$ with biologic therapy. The average year/ patient cost with NSAIDs alone would be EUR 381, with DMARDs only EUR 9,318 and, if only biological therapy was used, EUR 423 . Within the total number of patients, the average annual cost, including the possibility of combining these drugs, amounted to EUR 5,403

Conclusion: Including biological therapy in the care of patients with spondyloarthritis can increase up to 24 times the annual cost per patient. This increase is not only due to higher market value, it also relates to the need for more medical procedures and diagnostic follow-up tests.

References:

[1] Strömbeck, et al. Cost of Illness from the Public Payers' Perspective in Patients with Ankylosing Spondylitis in Rheumatological Care. J Rheumatol 2010;37;2348-2355.

Table 1

Table 1
\begin{tabular}{l|l|l|l}
\hline Distribution by therapy & \multicolumn{3}{l}{ Cost monotherapy treatments (EUR) } \\
\hline NSAIDs & $66 \%$ & Patient with NSAIDs & $\$ 381$ \\
\hline Biological Therapy & $57 \%$ & Patient with biological therapy & $\$ 9.318$ \\
\hline DMARDs & $33 \%$ & Patient with DMARDS & $\$ 423$ \\
\hline
\end{tabular}

Average patient $\infty$ st year $\$ 5,403$ EUR

Table 2

\begin{tabular}{|l|r|}
\hline \multicolumn{2}{|c|}{ Distribution of average annual cost per patient } \\
\hline Analgesic & $0,72 \%$ \\
\hline Biological & $94,2 \%$ \\
\hline Procedures & $1,32 \%$ \\
\hline Diagnosis & $3,12 \%$ \\
\hline For osteoporosis & $0,64 \%$ \\
\hline
\end{tabular}

Disclosure of Interests: None declared

DOI: 10.1136/annrheumdis-2020-eular.5143

\section{AB0635 HOW ARE NON STEROIDAL ANTI-INFLAMMATORY DRUGS (NSAID) PRESCRIBED IN AXIAL SPONDYLOARTHRITIS?}

K. Ben Abdelghani ${ }^{1}$, Y. Gzam ${ }^{1}$, A. Fazaa ${ }^{1}$, S. Miladi ${ }^{1}$, K. Ouenniche ${ }^{1}$, S. Kassab ${ }^{1}$, L. Souabni ${ }^{1}$, S. Chekili ${ }^{1}$, A. Laatar ${ }^{1} .{ }^{1}$ Mongi Slim Hospital, Rheumatology, Tunis, Tunisia

Background: For decades, NSAID have been used as the first-line drugs to treat axial spondyloarthritis (ax-SpA). However, the NSAID prescription strategy is not clearly detailed and it varies from one clinician to another.

Objectives: The aim of this study was to assess the NSAID prescription modalities adopted in ax-SpA and the differences between these modalities.

Methods: This is a descriptive study including 200 cases of ax-SpA fulfilling the ASAS 2009 criteria and diagnosed between January 2000 and October 2019. The demographic and clinical features of the ax-SpA were collected and the modalities of prescription of NSAID were retrospectively assessed.

Results: Our population consists of 138 men and 62 women with a mean age of $43,3 \pm 11,2$ years. The HLA B-27 antigen was present in $50,8 \%$ of cases. The ax-SpA was a pure axial form in $67 \%$ of patients, associated with peripheral arthritis, enthesitis and dactylitis in $19 \%, 21,5 \%$ and $1,5 \%$ respectively.

One hundred eighty patients $(90 \%)$ had been treated with NSAIDs. The NSAIDs used were: the Diclofenac $(57.5 \%)$, Indomethacin $(37.5 \%)$, Piroxicam $(36 \%)$, clecoxib (34\%), Naproxen (29.5\%) and ketoprofen (13\%). Seventy-three patients $(36.5 \%)$ had used at least 3 NSAIDs.

Among the 180 patients treated with NSAID, 88 patients $(48,8 \%)$ were treated with conventional synthetic DMARDs (csDMARDs) in association with NSAID: Salazopyrine (43,3\%) and Methotrexate (13,3\%). Seventy-one patients $(39,4 \%)$ had necessitated the use of anti-TNF alpha.

NSAIDs were used continuously in 115 patients $(63.8 \%)$ and the maximum dose of NSAIDs was used in 78 patients (43.3\%). By comparing patients who used maximum doses of NSAIDs and those who used NSAID continuously with other patients, we noticed that the use of biological treatments was more frequent in those groups ( $p=0,01$ and $p=0,004$ respectively).

In addition, while comparing the group of patients co-treated with csDMARDs with other patients treated with NSAID on monotherapy, we noted that this group of patients had more arthritis $(p<0,0001)$, enthesitis $(p=0,02)$, psoriasis $(p=0,04)$ and necessitated more biological treatments $(p=0,01)$.

Conclusion: Our results suggest that maximal doses and/or continuous prescription of NSAID were mainly used if there was no response to that treatment. The csDMARDs were more prescribed if there were peripheral manifestations or psoriatic arthritis and those forms were also more candidates to biological treatments.

\section{References:}

[1] Wang R, et al. Arthritis Rheumatol Hoboken NJ. 2019;

Disclosure of Interests: None declared

DOI: 10.1136/annrheumdis-2020-eular.6221

\section{$\mathrm{AB} 0636$ \\ MODALITIES OF PRESCRIPTION OF ANTI-TNF ALPHA IN AXIAL SPONDYLOARTHRITIS: ON MONOTHERAPY OR COMBINED WITH CONVENTIONAL SYNTHETIC DMARDS}

K. Ben Abdelghani ${ }^{1}$, Y. Gzam ${ }^{1}$, A. Fazaa ${ }^{1}$, S. Miladi ${ }^{1}$, K. Ouenniche ${ }^{1}$, S. Kassab ${ }^{1}$, L. Souabni ${ }^{1}$, S. Chekili ${ }^{1}$, A. Laatar ${ }^{1} .{ }^{1}$ Mongi Slim Hospital, Rheumatology, Tunis, Tunisia

Background: The advent of biologics targeting tumor necrosis factor-alpha (antiTNF alpha) has revolutionized the treatment of spondyloarthritis (SpA). Their association with conventional synthetic disease-modifying antirheumatic drugs (cs-DMARD), although effective and used in clinical practice for the treatment of peripheral rheumatic diseases, is not clearly assessed in axial spondyloarthritis (ax-SpA).

Objectives: The aim of this study was to assess the strategy of prescription of anti-TNF alpha in a population of ax-SpA and to compare patients treated with anti-TNF alpha on monotherapy with those who had combined therapy with cs-DMARDs.

Methods: This is a retrospective descriptive study including 85 cases of ax-SpA diagnosed between January 2000 and October 2019 and treated with anti-TNF alpha.

The clinical features, the erythrocyte sedimentation rate (ESR), the C-reactive protein (CRP), Bath ankylosing spondylitis disease activity index (BAS$\mathrm{DAI}$ ) and Bath ankylosing spondylitis functional index (BASFI) were compared between groups of anti-TNF alpha on monotherapy or combined therapy with csDMARDs.

Results: Of 85 ax-SpA, 67 were males (78,8\%) and the mean age was $44,4 \pm$ 10,9 years. The mean period of evolution was $12,3 \pm 9,1$ years and $52,2 \%$ of patients were HLA-B27 positive. The ax-SpA was a pure axial form in $74,1 \%$ of patients, associated with peripheral arthritis, enthesitis and dactylitis in $17,6 \%$, $17,6 \%$ and $1,2 \%$ respectively.

The ant-TNFs were administrated with a men delay of $78 \pm 70,8$ months. The anti-TNFs used were: Infliximab $(41,1 \%)$, Etanercept $(32,9 \%)$, Adalimumab $(23,5 \%)$ and Golimumab (2,3\%). Fifty-nine patients $(69,4 \%)$ were treated with anti-TNF alpha on monotherapy and 26 patients $(30,6 \%)$ had combined therapy. The csDMARDs prescribed were the Salazopyrine $(22,4 \%)$ and the Methotrexate (7,1\%).

While comparing the groups of anti-TNFs combined therapy and monotherapy, we noticed that the arthritis were present in $30,7 \%$ of patients from the group of combined therapy versus $11,8 \%$ of patients from the group of monotherapy $(p=0,03)$. The psoriasis also was more present in the group of combined therapy $(11,5 \%$ vs $1,6 \% ; p=0,04)$.

There was no statically significant difference between the two groups in the following parameters: age, gender, HLA B27, enthesitis, dactylitis, uveitis, inflammatory bowel diseases, ESR, CRP, BASDAI and BASFI.

Conclusion: Our results suggest that the concomitant use of csDMARDs with anti-TNFs is frequent in clinical practice in ax-SpA, but mainly justified by the presence of arthritis or psoriasis.

References:

[1] Engel-Nitz NM, et al. Rheumatol Ther. 2015;2(2):127-39.

Disclosure of Interests: None declared

DOI: 10.1136/annrheumdis-2020-eular.6242

\section{AB0637 EFFICACY OF COMEDICATION OF CONVENTIONAL SYNTHETIC DMARDS WITH TNF BLOCKERS IN PATIENTS WITH AXIAL SPONDYLOARTHRITIS}

K. Ben Abdelghani ${ }^{1}$, Y. Gzam ${ }^{1}$, A. Fazaa ${ }^{1}$, S. Miladi ${ }^{1}$, K. Ouenniche ${ }^{1}$, S. Kassab ${ }^{1}$, L. Souabni ${ }^{1}$, S. Chekili ${ }^{1}$, A. Laatar ${ }^{1} .{ }^{1}$ Mongi Slim Hospital, Rheumatology, Tunis, Tunisia

Background: Tumour necrosis factor blockers (anti-TNFs) are typically used in axial spondyloarthritis (ax-SpA) when the disease has not responded adequately to conventional therapy. However, the effects of the comedication conventional synthetic disease modifying antirheumatic drugs (csDMARDs) with anti-TNFs are inconclusive. 
Objectives: The aim of this study was to evaluate the efficacy of comedication csDMARD and anti-TNF compared with anti-TNFs on monotherapy.

Methods: A descriptive retrospective study including 85 patients with ax-SpA according to the criteria of the group ASAS on 2009 and having received antiTNFs between January 2000 and October 2019.

The patients were divided on two groups, those who had received combined therapy with cs-DMARDs and those who had received anti-TNFs on monotherapy. The response to treatment was assessed with the ASAS 40 response and partial remission at 3 and 6 months of treatment and was compared between the two groups.

Results: Our populations consists of 67 men and 18 women with a mean age of $44,4 \pm 10,9$ years. The mean period of evolution was $12,3 \pm 9,1$ years and $52,2 \%$ of patients were HLA-B27 positive. The ax-SpA was associated with peripheral arthritis, enthesitis and dactylitis in $17,6 \%, 17,6 \%$ and $1,2 \%$ respectively.

Fifty-nine patients $(69,4 \%)$ were treated with anti-TNF alpha on monotherapy and 26 patients $(30,6 \%)$ had combined therapy. The ASAS 40 response was achieved in $45,6 \%$ of patients at 3 months and $64,1 \%$ of them at 6 months of anti-TNFs treatment. Among them, 7,4\% had obtained partial remission at 3 months and $20,3 \%$ at 6 months of treatment.

There was statically significant difference between the two groups on the ASAS 40 response or the partial remission at 3 and 6 months of treatments.

Conclusion: The comedication therapy with csDMARDs does not influence the efficacy of anti-TNFs in ax-SpA patients suggesting no benefit in the concomitant use of these drugs in clinical practice.

References:

[1] Simone Det al. J Rheumatol Suppl. 2015;93:65-9.

Disclosure of Interests: None declared

DOI: 10.1136/annrheumdis-2020-eular.6320

\section{\begin{tabular}{|l|l}
\hline AB0638 & THE EFFECT OF VITAMIN D ON QUALITY OF LIFE AND
\end{tabular} SEVERITY OF PAIN IN PATIENTS WITH ANKYLOSING SPONDYLITE}

E. Komarova ${ }^{1}$, I. Blaginina ${ }^{1}$, N. Bludova ${ }^{1}$, P. Bakhtoyarov ${ }^{1}$, I. Pokryshka ${ }^{2}$. ${ }^{1}$ Lugansk State Medical University, Lugansk, Ukraine; ${ }^{2}$ Lugansk Republican Clinical Hospital, Lugansk, Ukraine

Background: The high incidence of ankylosing spondylite (AS) in people of working age, as well as the negative impact of the disease on the quality of life of patients, determine the need for adjuvants to reduce the severity of pain and thereby achieve the physical, psychological and emotional well-being of patients.

Objectives: To study the effect of vitamin $D$ (colecalciferol) on the quality of life and the severity of pain in patients with ankylosing spondylite.

Methods: The study included 69 patients with AS, who studied the quality of life indicators according to the Medical Outcomes Study Short Form (SF-36); pain syndrome and stiffness in the spine were assessed by a visual analogue scale by patients, and by a physician - by counting the number of painful joints (NPJ), the disease activity index (BASDAI) and the Functional Index (BASFI). All patients were receiving a basic therapy in a stable dose for at least 10 months. They were divided into 2 groups, comparable in age, disease activity; Group 1 ( $n=33$ ) additionally received colecalciferol $1500 \mathrm{ME}$ during 6 months of observation.

Results: At the end of the observation period when evaluating data on SF-36: in the 1st group, the physical health component has improved - the increase in physical functioning (PF) and bodily pain (BP) by $51.4 \%$ and $37.8 \%$ from the baseline; vital activity, psychological health, and social functioning due to emotional state have also increased by $37.6 \%, 33.4 \%$ and $42.5 \%$, respectively. In the 2nd group above mentioned parameters have not changed. In the 1st group the indexes of BASDAI and BASFI have decreased by $16 \%$ and $22 \%$ ( $p=0.0079, p=0.0022$, respectively), and their dynamics in the 2nd group were less significant ( $\mathrm{p}=0.013, \mathrm{p}=0.017$, respectively) Also, in patients of the 1st group have decreased the severity of morning stiffness and the pain in the spine a highly reliable $(p<0.001)$, and in the 2 nd group they were less significant ( $p=0.043, p=0.016$, respectively). Positive dynamics of NPJ in the 1st group was more significant $(p=0.003)$ than it was in the 2 nd group $(p=0.033)$.

Conclusion: In the group of patients treated with colecalciferol was noted improvement in indicators of quality of life (the parameters of the physical component of health, vitality and social functioning) and also more significant decrease of the intensity of pain and of morning stiffness duration, of NPJ, than in not received to colecalciferol patients. Inclusion of vitamin D in the comprehensive AS therapy promotes not only reduction the severity of the chronic pain manifestations, but also improves the quality of life of patients with this pathology.
Disclosure of Interests: None declared

DOI: 10.1136/annrheumdis-2020-eular.933

\section{AB0639 COHORT OF SPONDYLOARTHRITIS PATIENTS}

A. Castilla ${ }^{1}$, Á. García Martos ${ }^{2}$, L. Sala Icardo ${ }^{3}$, A. Prada-Ojeda ${ }^{3}$, L. Barrio Nogal $^{3}$, M. C. Ortega de la $\mathrm{O}^{4}$, C. Arconada ${ }^{4}$, L. González ${ }^{2}$, A. Diaz Oca ${ }^{5}$

P. Navarro6. ${ }^{1}$ University Hospital La Paz, Rheumatology, Madrid, Spain;

${ }^{2}$ Hospital del Tajo, Aranjuez, Spain; ${ }^{3}$ Hospital de Torrejón, Torrejon de Ardoz,

Spain; ${ }^{4}$ Hospital Universitario Infanta Elena, Valdemoro, Spain; ${ }^{5}$ Hospital

Universitario de Fuenlabrada, Fuenlabrada, Spain; ${ }^{5}$ Hospital Universitario de

Fuenlabrada, Fuenlabrada, Spain

Background: Spondyloarthropathies are a heterogeneous group of similar diseases, with interrelated clinical manifestations, such as Psoriasis Arthritis (APs) and Ankylosing Spondylitis (EA). There are different treatments for this group of pathologies.

Objectives: It is very important to differentiate between those that present predominantly axial (spine and/or sacroiliac) or peripheral involvement, since the response to treatment is very different.

In some clinical trials, secukinumab significantly improved versus placebo, the symptoms and signs, physical function and quality of life, however, at present, we do not have enough data from secukinumab in real clinical practice.

This is the real reason of this study: the use of secukinumab in clinical practice. Methods: Multicentric longitudinal observational study of 5 Hospitals in Madrid. Patients are over 18 years old and meet the following inclusion criteria: New York criteria for AS, ASAS for EA, CASPAR for APs, and all of them are with secukinumab or have received it.

We will evaluate the effectiveness rate as well as its confidence interval at $95 \%$. In addition, the effectiveness of secukinumab will be compared in the different pathologies by using $x^{2}$

Results: 72 patients were collected, 41 of them were women $(57,75 \%)$.

12 patients $(16,90 \%)$ had not received FAME before secukinumab and 22 patients $(33,99 \%)$ were naive to biological treatment.

In 4 patients, the reason for starting secukinumab was the patient's comorbidities, in 2 the adverse effects of previous treatment and in the rest, was the lack of efficacy of the previous treatment.

The patients were divided into 4 categories according to the level of DAS-28 or BASDAl, at the beginning of the treatment and the last recorded value, in: Absence of activity, mild, moderate and severe activity. Of the patients with data, they managed to improve the DAS- 28 score (change category) by $30,95 \%$, while only $4,76 \%$ worsened their score. With respect to BASDAI, of the total number of patients, only $3,03 \%$ worsened, while his score improved $27,27 \%$

According to baseline diagnosis, a greater improvement of the disease activity in peripheral $\operatorname{APs}(66,67 \%)$ and mixed $\operatorname{APs}(61,54 \%)$ is achieved.

Conclusion: In real clinical practice, treatment with secukinumab was effective in patients with spondyloarthritis, achieving improvement in previous activity rates. In this study, the most significant improvement was obtained in peripheral and mixed APs.

Disclosure of Interests: None declared

DOI: 10.1136/annrheumdis-2020-eular.5400

\section{AB0640 LONG-TERM EFFECTIVENESS AND DRUG SURVIVAL OF GOLIMUMAB IN PATIENTS AFFECTED BY PSORIATIC ARTHRITIS WITH CUTANEOUS INVOLVEMENT}

G. L. Fonti ${ }^{1}$, M. S. Chimenti ${ }^{1}$, A. D'antonio ${ }^{1}$, M. Teoli ${ }^{2}$, F. Caso ${ }^{3}$, L. Costa ${ }^{3}$, M. Tasso ${ }^{3}$, A. Ortolan ${ }^{4}$, M. Lorenzin ${ }^{4}$, P. Conigliaro ${ }^{1}$, P. Triggianese ${ }^{1}$, R. Scarpa ${ }^{3}$, R. Perricone ${ }^{1}$, R. Ramonda ${ }^{4} .{ }^{1}$ Rheumatology, Allergology and Clinical Immunology, Department of Systems Medicine, University of Rome Tor Vergata, Rome, Italy, Rome, Italy; ${ }^{2}$ University of Rome Tor Vergata, Dermatology Department, Rome, Italy; ${ }^{3}$ Rheumatology Unit, Department of Clinical Medicine and Surgery, School of Medicine and Surgery, University of Naples Federico II, Naples, Italy; ${ }^{4}$ Rheumatology Unit, Department of Medicine-DIMED, University of Padova, Padova, Italy

Background: Psoriatic arthritis (PsA) is a chronic immune-mediated disease associated with psoriasis ( $\mathrm{PsO}$ ). Overexpression of inflammatory cytokines such as tumor necrosis factor (TNF)-a plays a key role in the pathogenic mechanisms. Golimumab (GLM) is a fully human monoclonal antibody lgG1k neutralizing TNFa approved for PsA and PsO, but effectiveness evaluation in real life remains a crucial issue. 